ISSN 0103-9954

\title{
RESISTÊNCIA À FLEXÃO ESTÁTICA DE ALGUNS PAINÉIS AGLOMERADOS COMERCIALIZADOS NO RIO GRANDE DO SUL
}

\section{STATIC BENDING PROPERTIES OF SOME PARTICLEBOARD SOLD IN RIO GRANDE DO SUL}

\author{
Clovis Roberto Haselein ${ }^{1}$ Elio José Santini ${ }^{2}$ Rute Berger ${ }^{3}$ \\ Jackson Roberto Eleotério ${ }^{4}$ Luciano Schereen $^{3}$
}

\section{RESUMO}

Foram testados em flexão estática sete tipos de painéis aglomerados comercializados no RS. De cada tipo, foram retirados ao acaso cinco painéis e de cada um destes cortados três corpos de prova para os ensaios de flexão segundo a norma ASTM D1037-93 (1995). Os resultados indicam que os painéis enquadram-se nos critérios das normas norte-americanas de padronização. Para alguns tipos de painéis foi possível estabelecer modelos matemáticos relacionando módulos de elasticidade e de ruptura à massa específica.

Palavras-chave: flexão estática, chapas aglomeradas.

\begin{abstract}
Seven types of particleboard, sold in RS, were tested in static bending. From each type, five panels were taken randomly and three pieces were cut from each panel, according to the ASTM D1037-93 (1995) standards. The results indicate that the panels are in accordance with the US commerce standards. For a few type of panels it was possible to establish mathematical models relating modulus of elasticity and rupture to board density.
\end{abstract}

Key words: static bending, particleboard.

\section{INTRODUÇÃO}

Atualmente, as chapas aglomeradas produzidas no Brasil destinam-se, na sua grande maioria,

1. Engenheiro Florestal, PhD., Professor Adjunto do Departamento de Ciências Florestais, Centro de Ciências Rurais, Universidade Federal de Santa Maria, CEP 97105-900, Santa Maria (RS). haseleic@ccr.ufsm.br

2. Engenheiro Florestal, Dr., Professor Adjunto do Departamento de Ciências Florestais, Centro de Ciências Rurais, Universidade Federal de Santa Maria, CEP 97105-900, Santa Maria (RS). santinie@ccr.ufsm.br

3. Acadêmicos do Programa de Pós-Graduação em Engenharia Florestal, Centro de Ciências Rurais, Universidade Federal de Santa Maria, CEP 97105-900, Santa Maria (RS).

4. Engenheiro Florestal, MSc., Professor da Universidade Regional de Blumenau, Rua Mal. Rondon, 89, Bairro Salto do Norte, CEP 89065-200, Blumenau (SC). jreleote@ furb.br 
à indústria moveleira, para produção de dormitórios e cozinhas. Muita da antiga idéia do consumidor de tratar-se de um painel de baixa resistência, que "desmancha" em contato com a umidade, tem sido superada, apesar de ainda existir certa intolerância com este tipo de produto.

A demanda das fábricas de móveis do RS é suprida com chapas produzidas no próprio Estado, em outros estados da Região Sul e em outros países como, por exemplo, a Argentina. Isso porque a demanda no Brasil tem sido maior do que a oferta nos últimos anos, o que torna caro o painel aqui comercializado, quando comparado ao mercado internacional (BANCO NACIONAL DE DESENVOLVIMENTO ECONÔMICO E SOCIAL, 1998). Parte desse problema foi solucionado com a desvalorização cambial e os grandes investimentos feitos pelas indústrias do setor nos últimos anos. Por outro lado, a exportação direta desse produto normalmente não é comum a grandes distâncias, por tratar-se de produto de baixo preço. Com relação a esse tipo de material, normalmente são exportados produtos (móveis) acabados ou semi-acabados com maior valor agregado. A matéria-prima utilizada na fabricação de chapas aglomeradas é oriunda, na sua totalidade, de florestas plantadas de pinus e eucalipto, o que facilita a aceitação no mercado internacional. Por destinarem-se a interiores, a colagem é feita exclusivamente com resina uréica .

Os aglomerados podem ser comercializados "crus" ou revestidos. Originalmente, usava-se lâminas de madeira para essa finalidade. A escassez desse material, especialmente no sul do Brasil, levou a substituição por lâminas sintéticas, tais como Finish Foil (FF) e Baixa Pressão (BP). Essas conferem melhor acabamento ao aglomerado, não havendo a necessidade de custos adicionais com tratamento de superfície na indústria moveleira. FF é uma película de papel (liso ou impresso com padrões "madeirados") que é colada à chapa. Já BP é uma folha de papel impregnada com melamina que é fundida à chapa através de pressão e calor. Pela maior resistência à umidade, os últimos são utilizados por exemplo em cozinhas enquanto os primeiros são mais utilizados para a confecção de armários.

Uma das dificuldades que se observa atualmente é a inexistência de normas nacionais de comercialização. Para o mercado interno não há exigências mínimas de qualidade da chapa além daquela desejada pelo fabricante do móvel. Já para móveis exportados, as chapas devem atender às normas impostas pelo mercado alvo.

Os objetivos do presente trabalho são testar, em flexão estática, alguns tipos de painéis aglomerados comercializados no RS, comparar os resultados com normas internacionais e, dessa forma, contribuir para o estabelecimento de parâmetros mínimos de qualidade, visando a facilitar a seleção do painel pela indústria moveleira.

\section{MATERIAL E MÉTODOS}

Em duas fábricas de móveis do RS, foram coletadas sete tipo de chapas aglomeradas. De cada tipo, selecionou-se, ao acaso, cinco painéis utilizados na condução dos ensaios. Valendo-se de cada painel, foram confeccionados três corpos de prova no sentido do comprimento e três no sentido da largura para realização dos testes de flexão estática, exceto os painéis $\mathrm{X}, \mathrm{V}, \mathrm{C}$ e E que foram testados, apenas, no sentido do comprimento. 
Os tipos A, B, D, V, X (Tabela 1) são FF, sendo que os tipos D e V são "madeirados" e os demais lisos. Já os tipos C e E são BP e lisos. O índice C ou L, seguindo o tipo, refere-se a corpo de prova retirado no comprimento (direção de fabricação) ou na largura (direção perpendicular à fabricação) do painel. Já o índice "s" refere-se a painel sem-recobrimento (XC com recobrimento e $\mathrm{XCs}$, sem recobrimento).

Na própria indústria, os corpos de prova foram cortados com comprimento de $50 \mathrm{~mm}$ mais 24 vezes a espessura nominal do painel e $76 \mathrm{~mm}$ de largura e posteriormente, transportados ao Laboratório de Produtos Florestais da UFSM. Após o recebimento, foram colocados em câmara climatizada a $20^{\circ} \mathrm{C}$ e $65 \%$ de umidade relativa onde permaneceram até o equilíbrio. Nessas condições, os corpos de prova alcançaram um teor de umidade médio de $8 \%$, valor confirmado após os testes. Os ensaios foram realizados segundo as recomendações da AMERICAN SOCIETY FOR TESTING AND MATERIALS (1995). Os testes foram feitos em máquina universal de ensaios, hidráulica, com capacidade de aplicação de carga de 20 toneladas. $O$ vão utilizado foi de 24 vezes a espessura do corpo de prova, sendo a velocidade regulada para $7 \mathrm{~mm} /$ minuto. Para avaliar as propriedades dos painéis, determinaram-se os módulos de elasticidade (MOE) e de ruptura (MOR) à flexão bem como a massa específica aparente e o teor de umidade de cada peça.

\section{RESULTADOS E DISCUSSÃO}

Os resultados obtidos, nos ensaios dos diferentes tipos de painéis, encontram-se resumidos na Tabela 1. Pode-se notar que o painel XC, com recobrimento, diferiu estatisticamente em nível de $95 \%$ de probabilidade, do painel XCs, sem-recobrimento, tanto em MOE como em MOR.

TABELA 1: Valores Médios de Massa Específica, Teor de Umidade, Módulo de Elasticidade (MOE) e de Ruptura (MOR) determinados em corpos de prova retirados segundo o comprimento ou segundo a largura para os diferentes painéis de madeira.

\begin{tabular}{l|c|c|c|c}
\hline Painel & $\begin{array}{c}\text { Massa Específica } \\
\left(\mathrm{g} / \mathrm{cm}^{3}\right)\end{array}$ & $\begin{array}{c}\text { Teor de Umidade } \\
(\%)\end{array}$ & $\begin{array}{c}\text { MOE } \\
\left(\mathrm{kgf} / \mathrm{cm}^{2}\right)\end{array}$ & $\begin{array}{c}\text { MOR } \\
\left(\mathrm{kgf} / \mathrm{cm}^{2}\right)\end{array}$ \\
\hline XCs & 0,68 & - & $26200 \mathrm{a}$ & $168,34 \mathrm{a}$ \\
CC & 0,65 & 7,2 & $34800 \mathrm{~b}$ & $194,54 \mathrm{~b}$ \\
VC & 0,65 & - & $33000 \mathrm{~b}$ & $213,27 \mathrm{~b}$ \\
XC & 0,68 & - & $38550 \mathrm{~d}$ & $215,30 \mathrm{~b}$ \\
DL & 0,68 & 8,7 & $36100 \mathrm{c}$ & $246,18 \mathrm{c}$ \\
EC & 0,68 & 7,7 & $46800 \mathrm{~g}$ & $246,20 \mathrm{c}$ \\
AL & 0,75 & 8,0 & $39850 \mathrm{~d}$ & $273,29 \mathrm{~d}$ \\
DC & 0,68 & 8,7 & $45200 \mathrm{f}$ & $288,68 \mathrm{de}$ \\
BL & 0,75 & 8,0 & $41250 \mathrm{e}$ & $293,10 \mathrm{e}$ \\
AC & 0,75 & 8,0 & $44100 \mathrm{f}$ & $290,55 \mathrm{e}$ \\
BC & 0,75 & 8,0 & $44700 \mathrm{f}$ & $292,72 \mathrm{e}$ \\
\hline
\end{tabular}

Em que: A,B,C,D,E,V,X = tipos de painéis; $\mathrm{C}=$ Comprimento; $\mathrm{L}=$ Largura; $\mathrm{s}=$ sem recobrimento. Médias seguidas pela mesma letra, entre linhas, não diferem estatisticamente pelo teste LSD de Fisher ao nível de 5\% de probabilidade de erro. 
No sentido do comprimento, o painel D não diferiu estatisticamente, tanto em MOE como em MOR dos painéis $\mathrm{A}$ e $\mathrm{B}$, de maiores densidades. O painel D é constituído de madeira macia (conífera) e os demais são constituídos em grande parte (ou totalmente) com madeira de folhosa. No geral, os painéis apresentaram maiores propriedades no comprimento do que na largura, o que evidencia pequena orientação das partículas no sentido da formação, comportamento que pode ser considerado normal em muitos painéis de madeira constituídos de partículas ou fibras.

O coeficiente de variação encontrado para os testes foi de 6,33, 13,28 e 16,87\%, respectivamente, para os ensaios de massa específica, módulo de elasticidade e módulo de ruptura.

Comparando os valores determinados no presente estudo com os constantes da Tabela 2, pode-se observar que todos os painéis atendem às exigências das especificações da norma norteamericana (ANSI A 208.1-87, para chapas do Tipo 1, coladas com UF, da classe M (média massa específica, 0,60 a $\left.0,85 \mathrm{~g} / \mathrm{cm}^{3}\right)$.

TABELA 2: Valores mínimos exigidos pela norma norte-americana (AMERICAN NATIONAL STANDARDS INSTITUTE, 1987; US DEPARTMENT OF AGRICULTURE, 1987) para painéis colados com UF e de média massa específica.

\begin{tabular}{lcc}
\hline Tipo & MOR $\left(\mathrm{kgf} / \mathrm{cm}^{2}\right)$ & $\mathrm{MOE}\left(\mathrm{kgf} / \mathrm{cm}^{2}\right)$ \\
\hline ANSI 1-M-1 & 112 & 17600 \\
ANSI 1-M-2 & 148 & 22900 \\
ANSI 1-M-3 & 169 & 28200 \\
\hline
\end{tabular}

Em que: $\mathrm{M}=$ chapas de massa específica média, variando de $0,6 \mathrm{~g} / \mathrm{cm}^{3}$ (M-1) a $0,85 \mathrm{~g} / \mathrm{cm}^{3}$ (M-3) a $5 \%$ de umidade.

As Figuras 1 e 2 mostram que existe boa relação entre massa específica e MOE e MOR. No

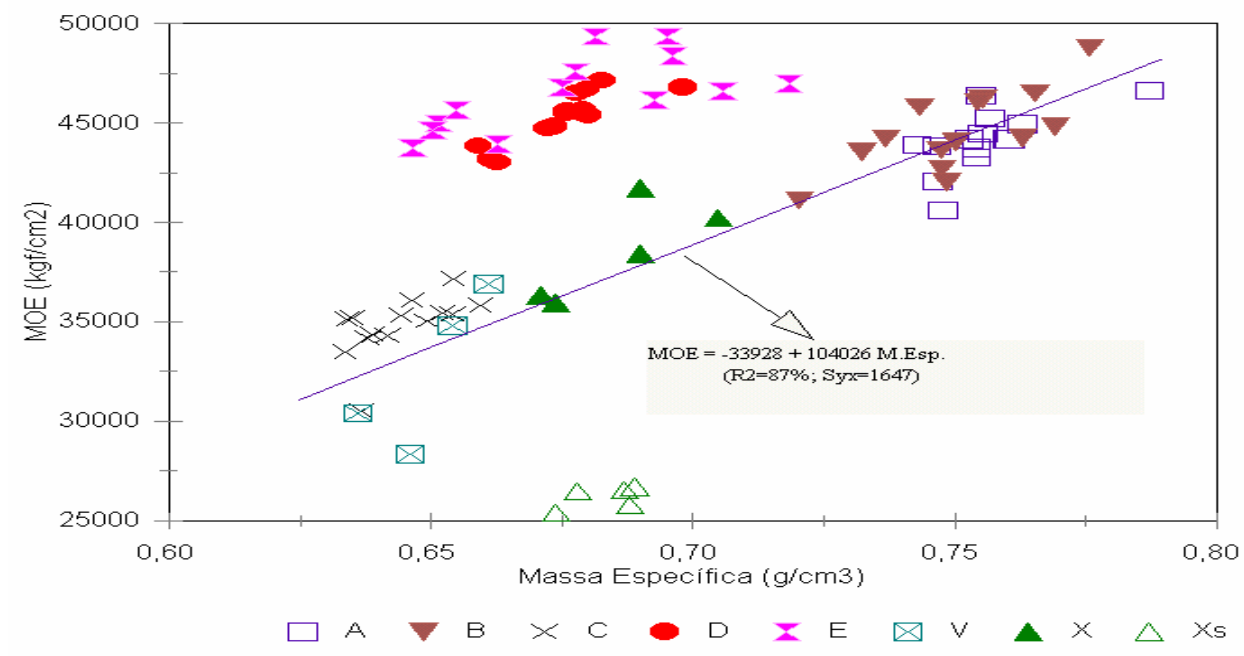

FIGURA 1: Módulo de elasticidade (MOE) no sentido do comprimento do painel em função da massa específica (equação de regressão ajustada apenas para os painéis tipo FF: A, $\mathrm{B}, \mathrm{V}$ e X). 


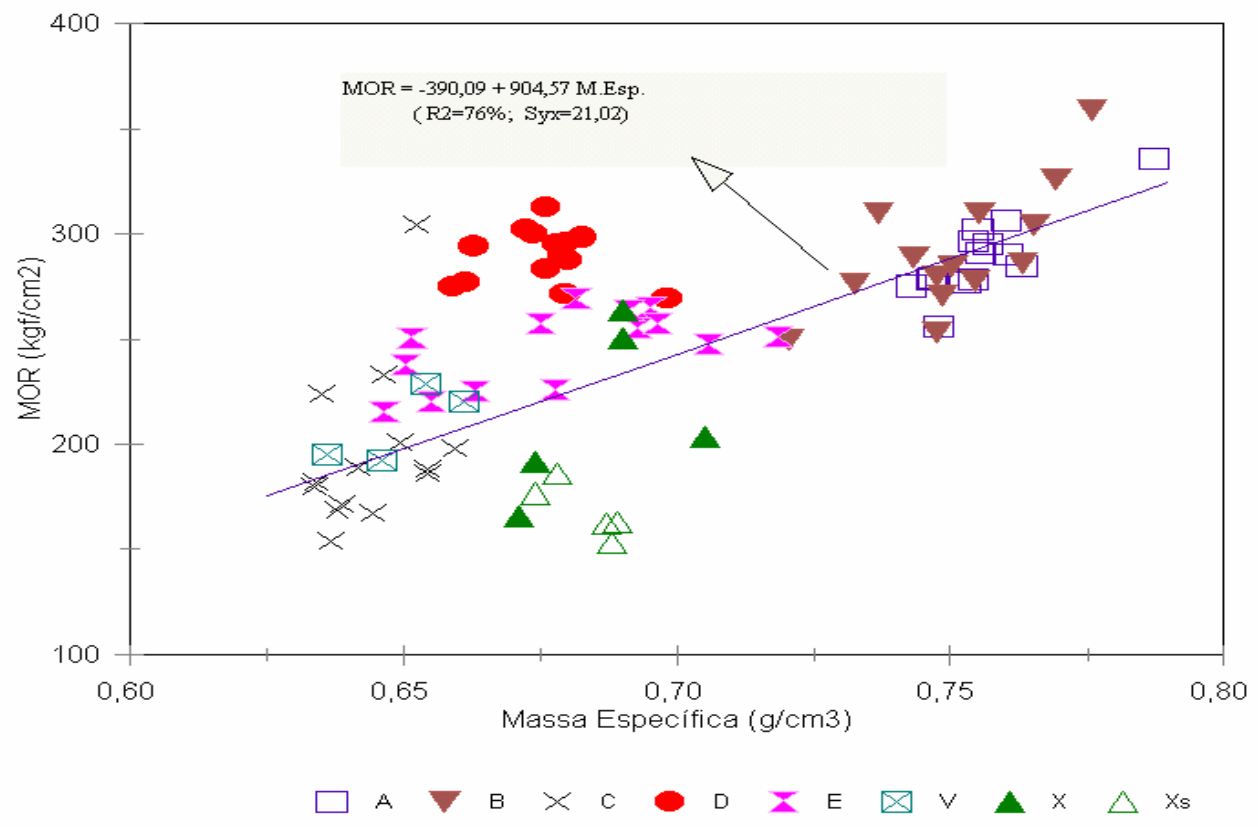

FIGURA 2: Módulo de ruptura (MOR) no sentido do comprimento do painel em função da massa específica (equação de regressão ajustada apenas para os painéis tipo FF: A, B, V e X).

entanto, outros fatores também podem ser importantes na definição da resistência do painel, como por exemplo a espécie de madeira usada na fabricação, a quantidade de resina e o tipo de recobrimento. Apesar de importante, estimativa da resistência à flexão somente pela massa específica é válida para um mesmo tipo de matéria-prima e, possivelmente, mesmas condições de fabricação. Por esse motivo, os modelos matemáticos estabelecidos para as estimativas excluem os painéis $\mathrm{C}, \mathrm{D}, \mathrm{E}$ e Xs (C e E possuem recobrimento BP, D é fabricado com madeira de conífera e Xs é cru). Apenas os painéis FF, produzidos com madeira de folhosas, foram incluídos nos modelos de regressão ajustados.

\section{CONCLUSÕES}

Considerando apenas a resistência à flexão estática, todos os painéis testados estão em conformidade com a norma norte-americana de comercialização e controle de qualidade. O painel Xcs atenderia a ANSI M1 e M2.

Os painéis $\mathrm{B}$, A e D são praticamente equivalentes desde que utilizados industrialmente com o maior vão no sentido do comprimento (direção de fabricação ou "machine direction"). O painel tipo D pode apresentar vantagens por ser mais leve.

Testes simples podem ser realizados pelas indústrias moveleiras visando à seleção do tipo de produto a adquirir. Outros testes podem e devem ser incorporados, tais como resistência ao arrancamento de parafusos, absorção d"água, entre outros. 


\section{REFERÊNCIAS BIBLIOGRÁFICAS}

AMERICAN NATIONAL STANDARDS INSTITUTE. Mat-formed wood particleboard. ANSI-A-208.187. New York, 1987.

AMERICAN SOCIETY FOR TESTING AND MATERIALS. Standard methods of evaluating the properties of wood-base fiber and particle panel materials. ASTM D1037-93, 1995.

BANCO NACIONAL DE DESENVOLVIMENTO ECONÔMICO E SOCIAL. Painéis de madeira aglomerada. 1998. Publicação Setorial: Produtos Florestais.

BODIG, J.; JAYNE, B.A. Mechanics of wood and wood composites. New York: Van Nostrand Reinhold Company, 1982. 712p.

U.S. DEPARTMENT OF AGRICULTURE. Wood handbook: wood as an Engineering Material. Washington, DC, 1987. Agriculture Handbook no. 72, U.S. Government Printing Office. 